\title{
Trastuzumab without chemotherapy in the adjuvant treatment of breast cancer: subgroup results from a large observational study
}

Peter Dall ${ }^{1 *}$, Thorsten Koch², Thomas Göhler ${ }^{3}$, Johannes Selbach ${ }^{4}$, Andreas Ammon ${ }^{5}$, Jochen Eggert ${ }^{6}$, Nidal Gazawi ${ }^{7}$, Daniela Rezek ${ }^{8}$, Arthur Wischnik ${ }^{9}$, Carsten Hielscher $^{10}$, Nicolas Schleif ${ }^{11}$, Ursula Cirrincione ${ }^{12}$, Axel Hinke ${ }^{12}$ and Gabriele Feisel-Schwickardi ${ }^{13}$

\begin{abstract}
Background: The topic of trastuzumab therapy without chemotherapy in early breast cancer (EBC) has been repeatedly discussed at international consensus meetings, but is compromised by the lack of solid evidence from clinical studies.

Methods: An observational study database of patients with EBC receiving trastuzumab-containing (neo)adjuvant therapy was screened to identify those patients who did not receive cytostatic agents.

Results: Of 3935 patients, 232 (6\%) were identified who received no chemotherapy, being characterized by older age, worse performance status, and/or less aggressive histology. Relapse-free survival in this cohort was 84\% (95\% confidence interval $[\mathrm{Cl}] 78-89 \%)$ at 3 years and $80 \%(95 \% \mathrm{Cl} 74-87 \%)$ at 5 years. However, these rates were significantly worse than those in the group of patients who received chemotherapy (hazard ratio 1.49; 95\% Cl 1.06-2.09; $P=0.022$ ). A similar pattern was observed for overall survival, with marginally non-significant inferiority in the group receiving no chemotherapy (hazard ratio 1.56; $95 \% \mathrm{Cl} 1.00-2.44 ; P=0.052$ ). Survival rates in patients receiving no chemotherapy were $93 \%(95 \% \mathrm{Cl} 88-97 \%)$ and $87 \%(95 \% \mathrm{Cl} 81-93 \%)$ at 3 and 5 years, respectively. These findings were confirmed by a propensity score analysis accounting for selection bias.

Conclusions: Trastuzumab plus chemotherapy should remain the preferred option in all patients with HER2-positive EBC with an indication for adjuvant treatment. However, a limited proportion of patients will need an alternative treatment approach, either because of contraindications or the patient's preference. In these selected patients, trastuzumab monotherapy, eventually combined with endocrine agents, might be a reasonable option offering favorable long-term outcomes by addressing the high-risk profile associated with HER2-positive disease.
\end{abstract}

Keywords: HER2-positive, Monotherapy, Overall survival, Propensity score analysis, Relapse-free survival

\section{Background}

For over a decade, the monoclonal antibody trastuzumab has been the cornerstone of adjuvant treatment for HER2-positive early breast cancer (EBC) $[1,2]$. Based on results from four large randomized trials [3-6], combined treatment with trastuzumab and chemotherapy (either as primary systemic or adjuvant treatment) is

\footnotetext{
* Correspondence: peter.dall@klinikum-lueneburg.de

${ }^{1}$ Department of Obstetrics and Gynaecology and Breast Cancer Center,

Städtisches Klinikum Lüneburg, Bögelstraße 1, D-21339 Lüneburg, Germany

Full list of author information is available at the end of the article
}

considered the standard of care in patients with this biologically aggressive subtype of breast cancer.

Although this evidence has led to unequivocal improvements in outcomes for the vast majority of patients with HER2-positive disease, the question remains as to whether there is a place for anti-HER2 therapy without chemotherapy in individually selected patients with EBC [7]. One major reason for this uncertainty is the fact that particular patient subgroups were underrepresented in the pivotal trials, including elderly patients $[8,9]$, those with significant concurrent disease, and those with small 
or low-risk tumors. In the latter subgroup, HER2-targeted therapy seems to be principally indicated, as several retrospective studies have shown that HER2 positivity leads to an unfavorable prognosis in patients with small cancers that are otherwise considered low risk [7, 10-13]. However, in view of the generally low rate of relapse events in these patients, chemotherapyinduced toxicity remains a major concern, leading to the question as to whether trastuzumab monotherapy is an adequate alternative option [7]. Hence, the issue of adjuvant trastuzumab monotherapy has repeatedly been discussed at international consensus meetings, resulting in weak recommendations and the recurrent request for randomized clinical trials. Unfortunately, such trials are difficult to perform due to the limited cohort size and the predictably low event rate. We therefore decided to approach this question within the framework of our database of about 4000 patients with EBC receiving trastuzumab.

This observational study [14] was started immediately after marketing authorization was received for Herceptin ${ }^{\mathrm{Tm}}$ (trastuzumab) treatment in EBC. Its purpose was to obtain real-world evidence on routine usage of trastuzumab in Germany. As this was a non-interventional study with no criteria concerning patient inclusion or treatment (apart from trastuzumab), the database included patients who were receiving trastuzumab without any cytotoxic treatment. This offered the opportunity to analyze outcomes in this subgroup and compare them with patients treated according to the standard approach, both by crude comparison and by application of a propensity score method to account for the assumed presence of selection bias.

\section{Methods}

\section{Patient population and methods of observation}

Details of the organizational and legal framework of this non-interventional study (Roche ML20315), the selection criteria for inclusion in the observation procedure, and the scope of the documentation have been described previously [14]. In general, patients were treated and their disease course was assessed according to routine practice at the treating institution. Findings were prospectively documented on standardized case report forms. Data on treatment toxicity were mainly collected throughout the duration of adjuvant therapy, i.e. up to 12 months. The study started in 2006 and database lock for the analyses presented here was October 2013.

\section{Endpoint evaluation and statistical analyses}

Relapse-free survival (RFS) and overall survival (OS) were calculated as the time between the baseline assessment before the first trastuzumab administration and the respective event. Surviving patients (without relapse for RFS) were censored at the last valid observation point. Event-related endpoints were analyzed using Kaplan-Meier methodology, with 95\% confidence intervals (CIs) for event-free proportions at specific time points. Univariate analyses comparing the treatment subgroups were performed using the log-rank test, while hazard ratios (HRs) with 95\% CIs were derived from Cox proportional hazards models [15]. In order to analyze the association between patient characteristics and the decision to withhold chemotherapy, $t$ tests, Fisher's exact tests, or appropriate trend tests for ordered categories were applied. All factors with an associated $P$-value $<0.1$ in univariate analysis were included in a multivariable logistic regression model.

Propensity score analysis [16], adjusting for selection bias when comparing the treatment subgroups with respect to RFS, was performed using the following prospectively planned steps: (1) covariate selection; (2) assessment of covariate balance before matching; (3) estimation of propensity scores by fitting a logistic regression model and matching procedure with a chosen sample size ratio of $1: 1 ;(4)$ assessment of covariate balance after propensity score matching; and (5) estimation of the treatment effect with a log-rank test, stratified by matched pairs, with RFS as the primary endpoint. As sensitivity analyses, unstratified methods were also applied because accounting for matching in time-to-event endpoints remains controversial $[17,18]$. The $R$ statistical software (R Foundation for Statistical Computing; https://www.r-project.org; Version 3.0) and its "matchit" package were used.

All statistical analyses were of an exploratory nature, with $P \leq 0.05$ termed significant, without any adjustments for multiplicity applied. All reported $P$-values are two-sided.

\section{Results \\ Baseline and treatment characteristics of patients with and without chemotherapy}

Between September 2006 and July 2011, a total of 3940 eligible patients with HER2-positive breast cancer were recruited, 3935 of whom could be unequivocally categorized into groups with $(n=3703 ; 94 \%)$ or without $(n=232 ; 6 \%)$ any sequential or concurrent (neo)adjuvant chemotherapy. Patient and tumor characteristics are shown in Table 1.

Patients receiving no chemotherapy were almost 3 years older on average $(P=0.0008)$ and more often presented with worse performance status $(P<0.0001)$ than those who received chemotherapy. In contrast, tumor-related characteristics such as TNM staging or hormone receptor status $(P=0.20)$ differed only marginally between the cohorts. Only poorly differentiated histology showed a moderate association with 
Table 1 Patient and tumor characteristics

\begin{tabular}{|c|c|c|c|}
\hline Characteristic & $\begin{array}{l}\text { Chemotherapy } \\
(n=3703)\end{array}$ & $\begin{array}{l}\text { No } \\
\text { chemotherapy } \\
(n=232)\end{array}$ & $P$-value \\
\hline \multicolumn{4}{|l|}{ Age, years } \\
\hline Mean (range) & $55.6(20-100)$ & $58.3(27-87)$ & \multirow[t]{4}{*}{0.0008} \\
\hline$<60, n(\%)$ & $2298(62)$ & $128(55)$ & \\
\hline $60-69, n(\%)$ & $971(26)$ & $58(25)$ & \\
\hline$\geq 70, n(\%)$ & $461(12)$ & $46(20)$ & \\
\hline \multicolumn{4}{|l|}{ ECOG performance status, $n(\%)$} \\
\hline 0 & $2301(63)$ & $108(47)$ & \multirow[t]{3}{*}{$<0.0001$} \\
\hline 1 & $1298(35)$ & $113(50)$ & \\
\hline $2-4$ & $61(2)$ & $7(3)$ & \\
\hline \multicolumn{4}{|l|}{ Primary tumor stage, $n(\%)$} \\
\hline $\mathrm{pT} 1 / \mathrm{is}$ & $1750(50)$ & $114(51)$ & \multirow[t]{2}{*}{0.73} \\
\hline pT2-4 & $1776(50)$ & $110(49)$ & \\
\hline \multicolumn{4}{|l|}{ Lymph node stage, $n$ (\%) } \\
\hline pNO & $1936(52)$ & $126(54)$ & \\
\hline pN1 & $1003(27)$ & $53(23)$ & \\
\hline $\mathrm{pN} 2$ & $411(11)$ & $28(12)$ & \\
\hline pN3 & $259(7)$ & $13(6)$ & \\
\hline NX & $82(2)$ & $12(5)$ & \\
\hline $\begin{array}{l}\text { No. of nodes involved, mean } \\
\pm \text { SD }\end{array}$ & $2.2 \pm 4.7$ & $2.3 \pm 5.1$ & 0.63 \\
\hline \multicolumn{4}{|l|}{ Grading, $n(\%)$} \\
\hline Grade 1/2 & $1731(47)$ & $123(55)$ & \multirow[t]{2}{*}{0.028} \\
\hline Grade 3 & $1927(53)$ & $101(45)$ & \\
\hline \multicolumn{4}{|l|}{ Hormone-receptor status, $n$ (\%) } \\
\hline ER positive & $2217(60)$ & $145(62)$ & \\
\hline PgR positive & $1857(50)$ & $133(57)$ & \\
\hline Either ER or PgR positive & $2332(63)$ & $156(67)$ & 0.20 \\
\hline \multicolumn{4}{|c|}{ Additional adjuvant treatment, $n(\%)$} \\
\hline Endocrine therapy & $2079(56)$ & $131(56)$ & 0.99 \\
\hline Radiotherapy & $2897(78)$ & $146(63)$ & $<0.0001$ \\
\hline
\end{tabular}

Total patient numbers may deviate from $n=3935$ because of missing values for some characteristics

ECOG Eastern Cooperative Oncology Group, ER estrogen receptor, $P g R$ progesterone receptor, SD standard deviation

administration of the more aggressive therapy approach $(P=0.028)$.

The administration of additional adjuvant endocrine treatment was equally common in both patient groups, but radiotherapy was more often omitted in patients who did not receive chemotherapy $(P<0.0001)$. No differences were detected between patients with and without chemotherapy with respect to trastuzumab exposure, with mean initial doses of 7.1 and $7.2 \mathrm{mg} / \mathrm{kg}$ body weight, mean number of cycles of 18.4 and 17.9, and mean duration of antibody therapy of 50.5 and 50 weeks, respectively.

\section{Multivariable analysis of treatment decision}

The significant parameters in the univariate analysis were included in a logistic regression model with the chosen treatment category as the dependent variable; all retained their independent level of association (Table 2). There is an obvious strong correlation between the decision to treat a patient with chemotherapy and the decision to use radiotherapy. Therefore a second regression analysis was done using a model that excluded the radiotherapy factor; it yielded almost unchanged results for the other factors. For the same reason, irradiation was not included in the propensity score procedure (see below).

\section{Trastuzumab-related toxicity}

Among the patients receiving chemotherapy, adverse reactions related to cardiac function (all severity grades) were reported in 154/3703 cases (4.2\%), with 93 (2.5\%) assessed as grade 2-4 (Common Terminology Criteria for Adverse Events V.3). The corresponding numbers in the cohort receiving no chemotherapy were $5 / 232(2.2 \%)$ and $4 / 232$ (1.7\%), respectively. The incidence of a pathological cardiac status during the baseline visit (detected by any type of cardiac monitoring) was similar between the two groups $(7 \%$ and $6 \%$ in those receiving and not receiving chemotherapy, respectively). At the end of adjuvant treatment the proportion was $8 \%$ in both groups. However, the general recommendations for heart function assessment were not followed in a considerable number of patients. The rate of patients having echocardiography was only around $60 \%$ per three-month time interval [14]. Other presumed adverse drug reactions of severity grade $3 / 4$ were rare in the monotherapy group: two cases of cardiac arrhythmia, two cases of dyspnea or other lung toxicity, and one patient with elevated liver enzymes.

\section{Long-term outcome: Crude analysis}

A total of 452 relapse-free survival events were observed before the database lock. In the chemotherapy group, the RFS rate was $90 \%$ (95\% CI 89-92\%) at 3 years and $83 \%$ (95\% CI $81-85 \%)$ at 5 years. The corresponding rates were distinctly lower in the cohort receiving no chemotherapy: $84 \%$ (95\% CI 78-89\%) and 80\% (95\% CI 74-87\%), respectively (Fig. 1a). The difference between treatment groups was statistically significant: HR 1.49 (95\% CI $1.06-2.09 ; P=0.022$ ). A similar pattern was observed for OS, although with only marginally non-significant inferiority for the group receiving no chemotherapy, based on a total of 248 reported deaths (HR 1.56; 95\% CI 1.00-2.44; $P=0.052$ ) (Fig. 1b). The 3- and 5 -year OS rates were $96 \%$ (95\% CI 96-97\%) and $90 \%$ (95\% CI 89-92\%) with chemotherapy, and 93\% (95\% CI 88-97\%) and 87\% (95\% CI 81-93\%) without chemotherapy, respectively. 
Table 2 Multivariable regression analysis of factors associated with treatment category

\begin{tabular}{lll}
\hline Factor $^{\mathrm{a}}$ & \multicolumn{2}{l}{ Odds ratio $^{\mathrm{b}}[P$-value $]$} \\
\cline { 2 - 3 } & $\begin{array}{l}\text { Multivariable } \\
\text { model } 1^{\mathrm{c}}\end{array}$ & $\begin{array}{l}\text { Multivariable } \\
\text { model } 2^{\mathrm{d}}\end{array}$ \\
\hline Age: $<65$ vs $\geq 65$ years & $1.51[P=0.0056]$ & $1.57[P=0.0020]$ \\
ECOG performance: 0 vs 1-4 & $1.80[P=0.00003]$ & $1.84[P=0.00001]$ \\
Grading: Grade 1/2 vs grade 3 & $0.77[P=0.062]$ & $0.77[P=0.058]$ \\
Radiotherapy: no vs yes & $0.53[P=0.00002]$ & - \\
\hline
\end{tabular}

ECOG, Eastern Cooperative Oncology Group

${ }^{\mathrm{a}}$ First group mentioned is reference; $-=$ not in model. ${ }^{\mathrm{b}} \mathrm{A}$ value $>1.0$ indicates a higher probability of receiving Herceptin treatment without chemotherapy, as compared to reference group. Including radiotherapy in the analysis.

${ }^{d}$ Excluding radiotherapy from the analysis

\section{Long-term outcome: Propensity score analysis}

Owing to the very high number of patients receiving chemotherapy, 204 monotherapy patients with a complete set of covariates available could be matched with 204 control patients, achieving perfectly balanced distributions for age ( $\geq 65$ years: $35 \%)$, T stage (pT2-4: $51 \%), \mathrm{N}$ stage ( $\mathrm{pN}+: 43 \%)$, grading (grade 3: $46 \%$ ), hormone receptor status (positive: 68\%), and performance status (ECOG 0: 48\%). As was expected, these proportions were very close to those described for the entire cohort without chemotherapy (Table 1).

Figure 2a shows the RFS results for the matched samples, with an HR of 1.41 (95\% CI $0.86-2.31 ; P=0.17)$ in the unstratified analysis, and HR 1.49 (95\% CI 0.88-2.52; $P=0.14)$ after stratification of the matched pairs. For OS, the corresponding results were HR 1.61 (95\% CI 0.81 -
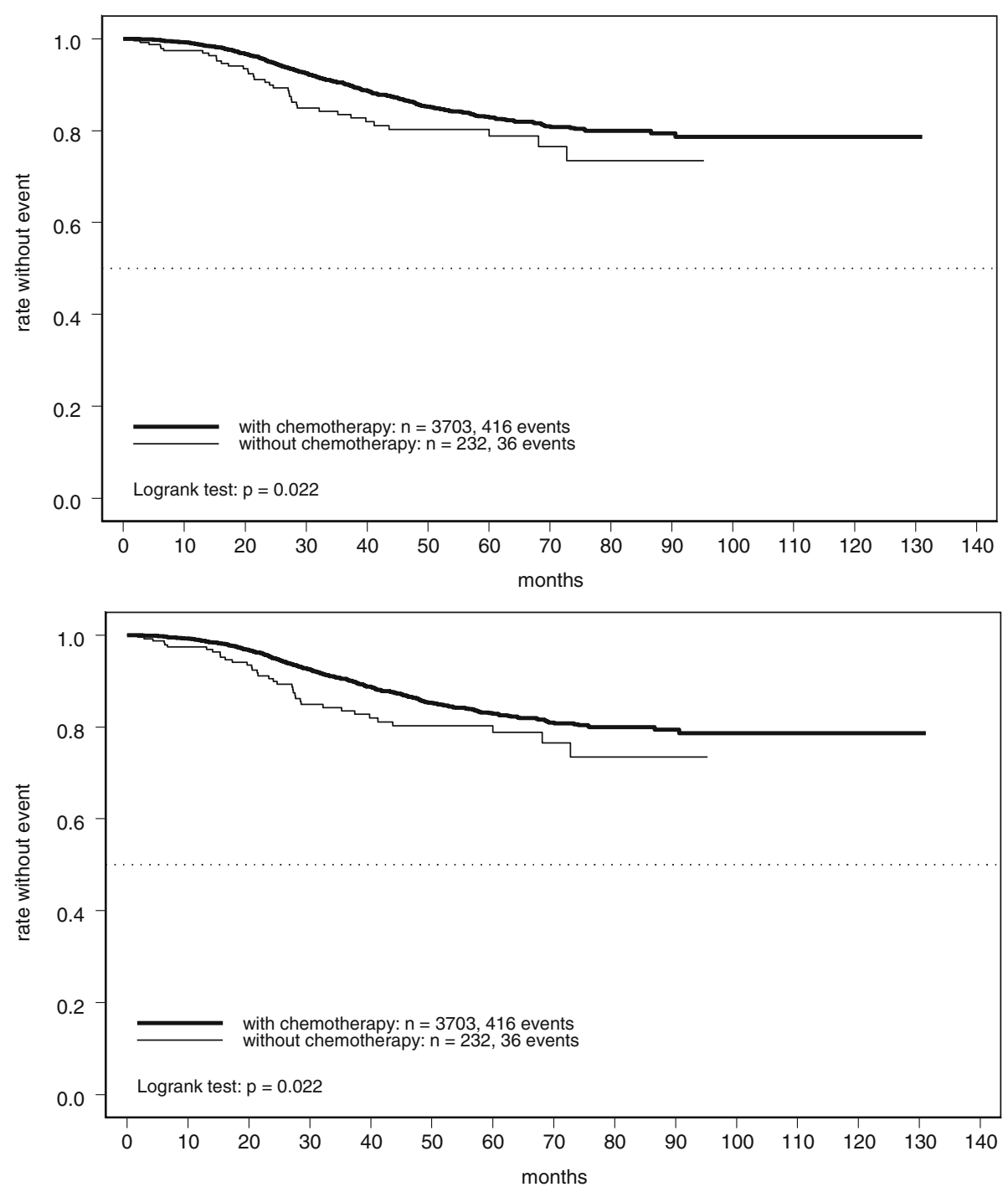

Fig. 1 Kaplan-Meier plots of (a) relapse-free survival and (b) overall survival in patients with early breast cancer receiving adjuvant trastuzumab with or without chemotherapy 

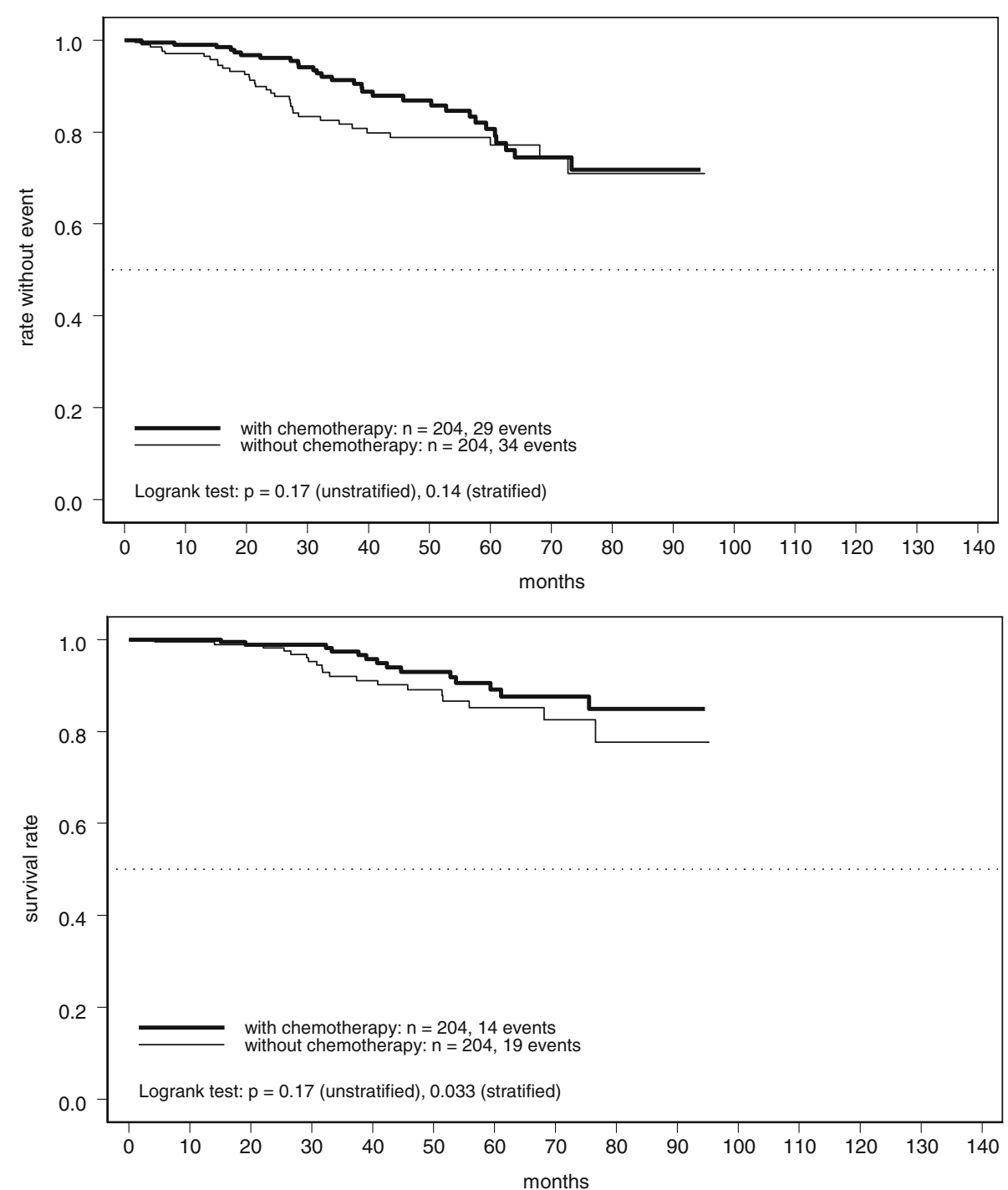

Fig. 2 Kaplan-Meier plots of (a) relapse-free survival and (b) overall survival in propensity score matched patients with early breast cancer receiving adjuvant trastuzumab with or without chemotherapy

3.22; $P=0.17)$ and HR 2.35 (95\% CI 1.05-5.25; $P=0.033)$

(Fig. 2b). The Kaplan-Meier curves and HRs did not differ qualitatively from the results of the crude analysis, suggesting that the superiority of the combined treatment is not an artifact caused by patient-selection bias. The wider CIs and larger $P$-values are an inevitable consequence of the limited number of observed events that remain after the matching procedure.

\section{Discussion}

In the initial treatment of patients with HER2-positive breast cancer, administration of a specific agent targeting this epitope is almost universally indicated, independent from tumor stage and patient-defined characteristics, such as age. Focusing on the volume of available evidence, current treatment guidelines almost exclusively recommend the use of combination regimens of HER2targeted therapy with either chemotherapy or a second targeted drug [2]. However, the efficacy of trastuzumab monotherapy was proven early in its clinical development program [7], even in heavily pretreated patients $[19,20]$. (Likewise, the addition of trastuzumab to endocrine agents in patients with hormone receptor-positive disease was shown to be beneficial [21].) The use of trastuzumab monotherapy in the adjuvant setting, which is frequently mentioned as an alternative option, can only rely on analogy to this evidence derived from the advanced or neo-adjuvant breast cancer setting [7].

In order to analyze the actual prevalence of this treatment approach in routine clinical practice and to gain insight into its clinical efficacy, we screened the database of 
our German observational study and found a small but considerable subgroup of patients who received trastuzumab without chemotherapy. This contrasts to other reallife studies from the Netherlands and UK, which reported no or minimal numbers of such cases [22, 23]. Our data show that the decision to take this treatment approach is clearly associated with expected characteristics, such as older age or worse general health status, and less aggressive histology, rather than tumor stage. These results from realworld data reflect findings for hypothetical cases presented to US oncologists in a survey asking for treatment recommendations in older adults with HER2-positive EBC [24].

Although these characteristics show distinct and statistically significant trends of selection, they do not reflect a clearly defined subpopulation of our total cohort. One major limitation of our study is that our documentation did not include any information on the individual reasons for not administering chemotherapy. Obtaining this information was prohibited for legal reasons, as including this question on the record form would have implied use of a treatment option that did not comply with the trastuzumab marketing authorization, which is not allowed for this type of observational study in Germany.

Nevertheless, we assume that a large proportion of our trastuzumab monotherapy cohort consists of patients who refused to be treated with cytotoxic chemotherapy. Publications on this topic are sparse [25], but in an American interview-based regional survey of 119 women who did not receive guideline-recommended adjuvant therapy, patient refusal was the reported reason for 31\% [26].

Long-term outcome after trastuzumab therapy without chemotherapy proved to be in an acceptable range, with an RFS rate of $80 \%$ after 5 years. However, in univariate comparisons against the cohort receiving chemotherapy, an advantage for the more aggressive approach was detected for both RFS and OS. We accounted for the presumed presence of selection bias by using a propensity score matching technique. This approach led to efficacy results (as reflected by HRs) that were comparable with the findings of the univariate analysis.

To gain an indication of the utility of trastuzumab monotherapy, we can compare our results against historical series of patients with HER2-positive breast cancer who did not receive adjuvant chemotherapy or trastuzumab. For example, a large cohort of 965 patients with small breast cancer tumors (T1a/bNOM0) from the MD Anderson Center had a 5-year RFS rate of only 77\% [12]. Indirect comparisons (with the usual caveats) against our RFS estimate of $80 \%$ suggest considerable benefit from trastuzumab monotherapy. This benefit becomes more obvious when considering that in our cohort approximately $50 \%$ were $\mathrm{T}$ stage $\geq 2$ and $41 \%$ were node positive, while proportions of hormone receptor positivity and endocrine treatment were roughly similar between the two studies.

\section{Conclusions}

In conclusion, trastuzumab plus chemotherapy should remain the preferred option in all patients with HER2-positive EBC and an indication for adjuvant treatment. However, a limited proportion of patients will need an alternative treatment strategy, either because of contraindications or patient preference. In these selected patients, trastuzumab monotherapy, eventually combined with endocrine therapy, might be a reasonable option offering favorable long-term outcomes by addressing the high-risk profile associated with HER2-positivity.

\section{Abbreviations}

Cl: Confidence interval; EBC: Early breast cancer; ECOG: Eastern Co-operative Oncology Group; ER: Estrogen receptor; HER2: Human epidermal growth factor receptor 2; HR: Hazard ratio; OS: Overall survival; PgR: Progesterone receptor; RFS: Relapse-free survival; SD: Standard deviation

\section{Acknowledgements \\ Not applicable.}

\section{Funding}

This study was sponsored and supported by Roche Pharma AG, GrenzachWyhlen, Germany. No grant number is applicable. Support for third-party writing assistance by WiSP Research Institute, Langenfeld, Germany was provided by Roche Pharma AG Germany.

\section{Availability of data and materials}

The data that support the findings of this study are available from Roche Pharma AG but restrictions apply to the availability of these data, which were used under license for the current study, and so are not publicly available. Data are however available from the authors upon reasonable request and with permission of Roche Pharma AG.

\section{Authors' contributions}

Conception/Design: PD, AH. Provision of study material or patients: PD, TK, $T G, J S, A A, J E, N G, D R, A W, C H, G F S$. Acquisition and assembly of data: PD, TK, $\mathrm{TG}, \mathrm{JS}, \mathrm{AA}, \mathrm{JE}, \mathrm{NG}, \mathrm{DR}, \mathrm{AW}, \mathrm{CH}, \mathrm{GFS}$. Data analysis and interpretation: PD, NS, UC, AH. Manuscript drafting: PD, NS, AH. Critical revision and final approval of manuscript: all authors. Accountable for all aspects of the work in ensuring that questions related to the accuracy or integrity of any part of the work are appropriately investigated and resolved: All authors read and approved the final manuscript.

\section{Ethics approval and consent to participate}

This was an observational study in which physicians' choices were guided by drug registration status and treatment guidelines (rather than the observation protocol). As the study was started prior to 2007, it was in agreement with the German FSA Codex and the German Arzneimittelgesetz Amendment 12, that there was no need/requirement for ethics committee approval or written informed consent. For non-interventional studies started in 2007 or later, the FSA Codex asks for submission to the ethics committee (recommended) and to the regulators [https://www.fsa-pharma.de/verhaltenskodizes/fachkreise/] (in German), accessed 07 August 2017]. Furthermore, in the European Union, clinical research has to be performed according to the Directive 2001/20/EC of the European Parliament and of the Council on the approximation of the laws, regulations and administrative provisions of the Member States relating to the implementation of good clinical practice in the conduct of clinical trials on medicinal products for human use dating from April 4th, 2001 (OJ. L121:34-44). This regulation differentiates between the requirements for "interventional" and "non-interventional" studies. This observational study clearly fulfils the criteria for "non-interventional" as defined in Article 2, c. 


\section{Competing interests}

Peter Dall: Roche Pharma AG, Novartis, Astra Zeneca (Honoraria received), Roche Pharma AG (Travel, accommodations or expenses); Carsten Hielscher: Roche Pharma AG, Celgene, Oncovis (Honoraria received); Pfizer, Oncovis (Travel, accommodations or expenses); Nicolas Schleif: Roche Pharma AG (Employment); Axel Hinke: Roche Pharma AG (Honoraria received). The other authors indicated no competing interests.

\section{Publisher's Note}

Springer Nature remains neutral with regard to jurisdictional claims in published maps and institutional affiliations.

\section{Author details}

'Department of Obstetrics and Gynaecology and Breast Cancer Center, Städtisches Klinikum Lüneburg, Bögelstraße 1, D-21339 Lüneburg, Germany. ${ }^{2}$ Breast Center, Klinikum Nürnberg Nord, Prof.-Ernst-Nathan-Str. 1, D-90419 Nürnberg, Germany. ${ }^{3}$ Onkozentrum Dresden/Freiberg, Leipziger Str. 118 D-01127 Dresden, Germany. ${ }^{4}$ Oncology Practice, Altmarkt 20 - 24, D-47166 Duisburg, Germany. ${ }^{5}$ Oncology Practice, Nikolausberger Weg 36, D-37073 Göttingen, Germany. ${ }^{6}$ Oncology Practice, Xantener Str. 40, D-47441 Moers, Germany. ${ }^{7}$ Gyneco-Oncology Practice, Lampestr. 1, D-04107 Leipzig, Germany. ${ }^{8}$ Gynecology Department, Marien-Hospital, Pastor-Janßen-Str. 8-38, D-46483 Wesel, Germany. 'Department of Gynecology, Klinikum Augsburg, Stenglinstr. 2, D-86156 Augsburg, Germany. ${ }^{10}$ Gyneco-Oncology Practice, Große Parower Str. 47 - 53, D-18435 Stralsund, Germany. ${ }^{11}$ Roche Pharma AG, Emil-Barell-Str. 1, D-79639 Grenzach-Wyhlen, Germany. ${ }^{12}$ WiSP Research Institute, Karl-Benz-Str. 1, D-40764 Langenfeld, Germany. ${ }^{13}$ Department of Obstetrics and Gynecology and Breast Cancer Center, Klinikum Kassel Mönchebergstr. 41 - 43, D-34125 Kassel, Germany.

\section{Received: 28 July 2017 Accepted: 28 November 2017}

\section{Published online: 08 January 2018}

\section{References}

1. Figueroa-Magalhaes MC, Jelovac D, Connolly RM, et al. Treatment of HER2positive breast cancer. Breast. 2014;23:128-36.

2. Coates AS, Winer EP, Goldhirsch A, et al. Tailoring therapies-improving the management of early breast cancer: St Gallen international expert consensus on the primary therapy of early breast cancer 2015. Ann Oncol. 2015;26:1533-46.

3. Smith I, Procter M, Gelber RD, et al. 2 year follow-up of trastuzumab after adjuvant chemotherapy in HER2-positive breast cancer: a randomized controlled trial. Lancet. 2007;369:29-36.

4. Perez EA, Romond EH, Suman VJ, et al. Four-year follow-up of trastuzumab plus adjuvant chemotherapy for operable human epidermal growth factor receptor 2-positive breast cancer: joint analysis of data from NCCTG N9831 and NSABP B-31. J Clin Oncol. 2011;29:3366-73.

5. Slamon D, Eiermann W, Robert N, et al. Adjuvant trastuzumab in HER2positive breast cancer. N Engl J Med. 2011;365:1273-83.

6. Moja L, Tagliabue $L$, Balduzzi $S$, et al. Trastuzumab containing regimens for early breast cancer. Cochrane Database Syst Rev. 2012;4:CD006243.

7. Constantinidou A, Smith I. Is there a case for anti-HER2 therapy without chemotherapy in early breast cancer? Breast. 2011;20(Suppl. 3):S158-61.

8. Brollo J, Curigliano G, Disalvatore D, et al. Adjuvant trastuzumab in elderly with HER2-positive breast cancer: a systematic review of randomized controlled trials. Cancer Treat Rev. 2013;39:44-50.

9. Molina-Garrido MJ, Guillen-Ponce C, Mora-Rufete A. Targeted anti-HER2 cancer therapy in elderly women diagnosed with breast cancer. Anti Cancer Agents Med Chem. 2014;14:639-45

10. Tovey SM, Brown S, Doughty JC, et al. Poor survival outcomes in HER2positive breast cancer patients with low-grade, node-negative tumours. $\mathrm{Br}$ J Cancer. 2009;100:680-3.

11. Joensuu $\mathrm{H}$, Isola J, Lundin $\mathrm{M}$, et al. Amplification of erbB2 and erbB2 expression are superior to estrogen receptor status as risk factors for distant recurrence in pT1N0M0 breast cancer: a nationwide population-based study. Clin Cancer Res. 2003;9:923-30.

12. Gonzalez-Angulo AM, Litton JK, Broglio KR, et al. High risk of recurrence for patients with breast cancer who have human epidermal growth factor receptor 2-positive, node-negative tumors $1 \mathrm{~cm}$ or smaller. J Clin Oncol. 2009;27:5700-6.
13. Curigliano G, Viale G, Bagnardi V, et al. Clinical relevance of HER2 overexpression/amplification in patients with small tumor size and nodenegative breast cancer. J Clin Oncol. 2009;27:5693-9.

14. Dall $P$, Koch $T$, Göhler $T$, et al. Trastuzumab in HER2 positive early breast cancer - results of a prospective, non-interventional study on routine treatment between 2006 and 2012 in Germany. Oncologist. 2017;22:131-8.

15. Cox DR. Regression models and life tables. J Roy Stat Soc B. 1972;34:187-202.

16. Austin PC. An introduction to propensity score methods for reducing the effects of confounding in observational studies. Multivariate Behav Res. 2011:46:399-424.

17. Williamson EJ, Forbes A. Introduction to propensity scores. Respirology. 2014;19:625-35.

18. Austin PC. The use of propensity score methods with survival or time-toevent outcomes: reporting measures of effect similar to those used in randomized experiments. Stat Med. 2014;33:1242-58.

19. Cobleigh MA, Vogel CL, Tripathy $D$, et al. Multinational study of the efficacy and safety of humanized anti-HER2 monoclonal antibody in women who have HER2-overexpressing metastatic breast cancer that has progressed after chemotherapy for metastatic disease. J Clin Oncol. 1999;17:2639-48.

20. Clemens M, Eidtmann H, Nitz U, et al. Trastuzumab single-drug therapy after failure of cytotoxic treatment for metastatic breast cancer. Onkologie. 2010; 33:425-30.

21. Kaufman B, Mackey JR, Clemens MR, et al. Trastuzumab plus anastrozole versus anastrozole alone for the treatment of postmenopausal women with human epidermal growth factor receptor 2-positive, hormone receptorpositive metastatic breast cancer: results from the randomized phase III TAnDEM study. J Clin Oncol. 2009;27:5529-37.

22. Seferina SC, Lobbezoo DJ, de Boer M, et al. Real-life use and effectiveness of adjuvant trastuzumab in early breast cancer patients: a study of the Southeast Netherlands breast cancer consortium. Oncologist. 2015;20:856-63.

23. Webster RM, Abraham J, Palaniappan N, et al. Exploring the use and impact of adjuvant trastuzumab for HER2-positive breast cancer patients in a large UK cancer network. Do the results of international clinical trials translate into a similar benefit for patients in south East Wales? Br J Cancer. 2012;106:32-8.

24. Hurria A, Wong FL, Pal S, et al. Perspectives and attitudes on the use of adjuvant chemotherapy and trastuzumab in older adults with HER-2+ breast cancer: a survey of oncologists. Oncologist. 2009;14:883-90.

25. Schuppert I. Die Behandlung von Brustkrebs ohne Chemo- und Strahlentherapie - Warum erkrankte Frauen diese Entscheidung treffen und wie das soziale Umfeld reagiert. Thesis, Frankfurt/Oder 2011. https://www. praxisklinikbonn.de/docs/Masterarbeit__Schuppert.pdf (accessed 27-10-2016).

26. Bickell NA, LePar F, Wang JJ, Leventhal H. Lost opportunities: physicians' reasons and disparities in breast cancer treatment. J Clin Oncol. 2007;18: 2516-21.

\section{Submit your next manuscript to BioMed Central and we will help you at every step:}

- We accept pre-submission inquiries

- Our selector tool helps you to find the most relevant journal

- We provide round the clock customer support

- Convenient online submission

- Thorough peer review

- Inclusion in PubMed and all major indexing services

- Maximum visibility for your research

Submit your manuscript at www.biomedcentral.com/submit
) Biomed Central 